\title{
Masers and star formation
}

\author{
Vincent L. Fish \\ Jansky Fellow, National Radio Astronomy Observatory, 1003 Lopezville Rd., Socorro, NM \\ 87801, USA \\ email: vfish@nrao.edu
}

\begin{abstract}
Recent observational and theoretical advances concerning astronomical masers in star forming regions are reviewed. Major masing species are considered individually and in combination. Key results are summarized with emphasis on present science and future prospects.
\end{abstract}

Keywords. masers - stars: formation - radio lines: ISM — ISM: molecules — ISM: jets and outflows - ISM: kinematics and dynamics

\section{Introduction}

This review summarizes maser results pertinent to star formation appearing in the literature since the last maser meeting (IAU Symposium 206). References are drawn from recent literature when possible.

\section{Masing species}

\subsection{Water $\left(\mathrm{H}_{2} \mathrm{O}\right)$}

The $22.235 \mathrm{GHz}$ water line is the predominant water maser line. Masers in this transition are very bright, easily observable, and inverted under a wide range of conditions (e.g., Babkovskaia \& Poutanen 2004). Several millimeter and submillimeter transitions of water are also seen as masers. Discussion of these transitions can be found in the section of these proceedings devoted to millimeter and submillimeter masers.

Water masers are frequently seen in outflows from both high-mass and low-mass YSOs (Honma et al. 2005; Moscadelli, Cesaroni, \& Rioja 2005; Goddi \& Moscadelli 2006; Moscadelli et al. 2006). These jets are seen in deceleration (Imai et al. 2002) and often have substructure on AU scales (Torrelles et al. 2003; Furuya et al. 2005; Uscanga et al. 2005).

Water masers are sometimes believed to trace disks as well as outflows (Seth, Greenhill, \& Holder 2002; Gallimore et al. 2003), possibly excited by an expanding shock wave. Indeed, shocks likely are responsible for arc-like maser distributions (Honma et al. 2004) and may excite masers in accreting material as well (Menten \& van der Tak 2004). Water masers appear in Bok globules (Gómez et al. 2006), likely tracing bipolar molecular outflows (de Gregorio-Monsalvo et al. 2006), as well as bright rimmed clouds (Valdettaro et al. 2005), again likely associated with outflows (Urquhart et al. 2006). The location of water masers near the ionization front of large $\mathrm{H}$ II regions provides evidence in support of triggered star formation (Healy, Hester, \& Claussen 2004). The common thread of all these environments is the existence of energetic shocks, which fits with conventional wisdom regarding water maser pumping.

Despite the small Zeeman splitting coefficient of water, line-of-sight magnetic fields of tens to hundreds of milligauss have been measured in star forming regions via water maser Zeeman splitting (Sarma et al. 2002; Vlemmings et al. 2006), although direct interpretation of the Stokes $\mathrm{V}$ profile as a magnetic field strength may be in error by up 
to a factor of two depending on local velocity and magnetic field gradients (Vlemmings 2006). Linear polarization observations of water masers can provide information on the orientation of the magnetic field in the plane of the sky. The hourglass morphology of the magnetic field in W3 IRS 5 appears to be due to the processes of collapse rather than a result of the outflow traced by water masers (Imai et al. 2003). Further interpretation of water maser polarization can be found in the review by Wouter Vlemmings.

Water masers have a fractal distribution over 4 orders of magnitude in spatial scale, possibly indicating that they appear at the turbulent dissipation scale (Strelnitski et al. 2002; Ripman \& Strelnitski 2006; Strelnitski et al. 2007). Turbulence may also be responsible for variability, including changes in the line-of-sight velocity of individual components, of the water masers in some sources (Lekht et al. 2006a). Larger-scale variations may contribute as well, such as changes in outflow parameters or cyclic variability of the central star (Pashchenko \& Lekht 2005; Lekht et al. 2006b). An ordered structure is inferred in W31(2) from successive flaring of features at different velocities (Lekht, Munitsyn, \& Tolmachev 2005).

\subsection{Methanol $\left(\mathrm{CH}_{3} \mathrm{OH}\right)$}

Methanol masers divide into two categories, known as class I and class II, based on their propensity for certain transitions to produce masers while others are seen in absorption. Traditionally, class I and class II masers do not mix (e.g., Ellingsen 2005); however, finetuned conditions may rarely excite lines from both classes simultaneously, as appears to be the case in OMC-1 (Voronkov et al. 2005). For purposes of this review, class I and class II methanol masers will be treated separately. Improved laboratory data for rest frequencies have been obtained for many methanol transitions in both classes (Müller, Menten, \& Mäder 2004).

\subsubsection{Class $I$}

Class I masers are primarily collisionally pumped. They are typically found in younger sources that are Class II masers and may trace distant parts of outflows interacting with dense molecular gas (Beuther et al. 2005; Ellingsen 2006). Comparison of interferometric maps of the 9.9 and $104.3 \mathrm{GHz}$ transitions with $\mathrm{H}_{2}$ data confirms the outflow association in IRAS 16547-4247 (Voronkov et al. 2006). Linear polarization suggests that Class I masers may appear in oblique shocks parallel to the outflow axis and perpendicular to the magnetic field in OMC-2 (Wiesemeyer, Thum, \& Walmsley 2004), although interpretation of methanol polarization may be complicated (e.g., Elitzur 2002).

Many different Class I transitions have been observed. Weak masers at 84.5 and $95.2 \mathrm{GHz}$ to the southwest of $\mathrm{W} 3(\mathrm{OH})$ provide strong evidence in support of collisional pumping and allow for physical conditions to be inferred (Sutton et al. 2004). The latter transition is commonly seen as a maser in both Class I and Class II sources (Minier \& Booth 2002). Strong $36 \mathrm{GHz}$ maser emission is believed to be an indicator of an early evolutionary stage, as may line ratios of other transitions (Gillis, Pratap, \& Strelnitski 2005; Hoffmann, Pratap, \& Strelnitski 2006). The intensity ratio of highly-excited 146.6 and $156.8 \mathrm{GHz}$ methanol masers may be a sensitive probe of density and temperature in masing regions (Lemonias, Strelnitski, \& Pratap 2006). Short-timescale variability is seen in the 44 and 146.6 GHz transitions (Pratap, Hoffmann, \& Strelnitski 2006).

\subsubsection{Class $I I$}

The key Class II maser transitions are at 6668 and $12178 \mathrm{MHz}$. Class II masers are often found in an earlier evolutionary stage than ultracompact (UC) H II regions (e.g., Minier et al. 2005), but observations of methanol masers cospatial with both millimeter 
and centimeter continuum emission indicate that Class II masers appear over a wide range of evolutionary stages (Pestalozzi et al. 2006). While the lower stellar mass limit for methanol masers is still a subject of research, $6.7 \mathrm{GHz}$ masers do not appear below approximately 3 solar masses (Minier et al. 2003).

Linear structures of masers with organized velocity structures have led some to conclude that methanol masers often trace edge-on disks (Norris et al. 1993, 1998). Observations of maser proper motions (Minier et al. 2000), shocked $\mathrm{H}_{2}$ (De Buizer 2003), and $\mathrm{SiO}$ (De Buizer et al. 2006) indicate that, in the majority of cases at least, methanol masers are aligned with an outflow, not a disk. These structures may be explained by propagation of a shock front into a region with large-scale velocity structure, such as rotation (Dodson, Ojha, \& Ellingsen 2004). Disk candidate sources remain (Slysh et al. 2002b; Pestalozzi et al. 2004; Pillai et al. 2006), although these, too, may turn out to be associated with outflows when studied at high resolution in the mid infrared (e.g., De Buizer \& Minier 2005). The conclusion to be drawn is that a linear distribution of masers with a velocity gradient does not by itself present convincing evidence that the masers trace an edge-on disk. An intriguing variant is the possibility of methanol masers tracing a face-on disk in G23.657-0.127 (Bartkiewicz, Szymczak, \& van Langevelde 2005).

There is evidence to support the hypothesis that most methanol masers are tracing shocked regions, often in the presence of outflows. Methanol masers appear preferentially near radio sources with a spectral index indicative of an outflow (Zapata et al. 2006). Midinfrared images of some sources indicate that masers are found along the shocked material on the surface of an outflow cavity (De Buizer 2006, 2007). In some sources, methanol masers appear near but offset from UCH II regions, suggesting that they appear in the shocked molecular gas outside the ionization front, similar to hydroxyl masers (Phillips \& van Langevelde 2005).

The $6.7 \mathrm{GHz}$ transition is a popular line for Galactic maser surveys. Several surveys were reported on during IAU Symposium 206. Details of the Arecibo and Parkes multibeam $6.7 \mathrm{GHz}$ surveys can be found in the section of these proceedings devoted to Galactic maser surveys. Unsurprisingly, their distribution correlates well with Galactic structure (Pestalozzi, Minier, \& Booth 2005; Pestalozzi et al. 2007). The 12.2 GHz line, when it occurs, is almost always weaker than $6.7 \mathrm{GHz}$ emission (Błaszkiewicz \& Kus 2004). Both lines have also been the subject of monitoring studies (e.g., Goedhart, Gaylard, \& van der Walt 2005), which find variability in a large fraction of sources including periodic variability and a time delay between features possibly due to light travel time (Goedhart, Gaylard, \& van der Walt 2005; Goedhart et al. 2005). Based on comparison of spectra over a period of a decade, the lifetime of an individual $6.7 \mathrm{GHz}$ maser feature is about 150 years (Ellingsen 2007), while the lifetime of the $6.7 \mathrm{GHz}$ maser phase in a source is a few $\times 10^{4}$ years (Codella et al. 2004; van der Walt 2005), similar to the lifetime of the $\mathrm{OH}$ maser phase (e.g., Fish \& Reid 2006).

Numerous other Class II transitions have been observed. New maser sources have been found in rare transitions at 85.5, 86.6, and 107.0 GHz (Minier \& Booth 2002; Ellingsen et al. 2003) and a torsionally-excited line at $44.9 \mathrm{GHz}$ (Voronkov, Austin, \& Sobolev 2002). Several weak maser lines near $165 \mathrm{GHz}$ have also been detected (Salii \& Sobolev 2006). Emission in the $19.9 \mathrm{GHz}$ transition is usually weak and correlates well with $6035 \mathrm{MHz} \mathrm{OH}$ masers (Ellingsen et al. 2004). A search for $23.1 \mathrm{GHz}$ emission resulted in no new detections beyond the previously known maser in NGC 6334F (Cragg et al. 2004). Observations of these less common methanol maser transitions can help constrain physical parameters in maser models. Improved collisional rate data has also allowed refinement of methanol models, which slightly affects predicted excitation conditions and 
brightness temperatures but not which transitions are expected to produce detectable Class II masers (Cragg, Sobolev, \& Godfrey 2005).

\subsection{Hydroxyl $(\mathrm{OH})$}

Hydroxyl masers are usually studied in sources with associated UCH II regions (e.g., Fish et al. 2005) but are also found toward less evolved massive protostellar objects (Edris, Fuller, \& Cohen 2007). A different class of $\mathrm{OH}$ masers is seen at the ends of the jet in the W3 TW object (Argon, Reid, \& Menten 2003).

Hydroxyl masers around more evolved sources are usually seen in expansion (sometimes very rapid; see Stark et al. 2007) ahead of the ionization front of a UCH II region (Fish \& Reid 2006). Sometimes the masers appear to trace a molecular disk or torus (Slysh et al. 2002a; Hutawarakorn \& Cohen 2005; Edris et al. 2005; Nammahachak et al. 2006). Masers are often seen along arcs or filaments (Cohen et al. 2006), with extended filamentary emission especially common at $4.7 \mathrm{GHz}$ (Palmer, Goss, \& Devine 2003).

Multitransition overlaps are of special interest because of their ability to constrain physical conditions in models. The $4765 \mathrm{MHz}$ line is observed to be the strongest line of the $6 \mathrm{~cm}$ triplet but is usually only weakly inverted and often spatially extended (e.g., Palmer, Goss, \& Whiteoak 2004; Harvey-Smith \& Cohen 2005). A histogram of $18 \mathrm{~cm}$ emission resembles $4.7 \mathrm{GHz}$ lineshapes in W49A, suggesting that the high-gain $18 \mathrm{~cm}$ emission and low-gain $6 \mathrm{~cm}$ emission have similar velocity distributions, even if the $4660 \mathrm{MHz}$ emission is spatially separate (Palmer \& Goss 2005). Much is made of overlaps between 4765 and $1720 \mathrm{MHz}$ masers (Palmer et al. 2003; Niezurawska et al. 2004, 2005). It should be noted that $6035 \mathrm{MHz}$ maser emission correlates more strongly with $4765 \mathrm{MHz}$ than does $1720 \mathrm{MHz}$, even if the velocities do not always agree (Dodson \& Ellingsen 2002; Smits 2003). Masers in the $1720 \mathrm{MHz}$ transition also appear to correlate with 1665 and $6035 \mathrm{MHz} \mathrm{OH}$ masers and $6.7 \mathrm{GHz}$ methanol masers, at least to arcsecond accuracy (Caswell 2004a). Masers in the $6030 \mathrm{MHz}$ transition are almost always accompanied by stronger emission at $6035 \mathrm{MHz}$ (Caswell 2003), with excellent spatial coincidence and agreement of magnetic field strengths with each other and with $1665 \mathrm{MHz}$ masers (Etoka, Cohen, \& Gray 2005). Masers in the highly-excited $13441 \mathrm{MHz}$ transition are rare but are always accompanied by $6035 \mathrm{MHz}$ masers at the same velocity, although the intensities in the two transitions do not show a high degree of correlation (Baudry \& Desmurs 2002; Caswell 2004c). The ground-state, satellite line transitions at 1612 and $1720 \mathrm{MHz}$ are usually conjugate with respect to absorption and maser emission (Szymczak \& Gérard 2004). Sources do exist in which both transitions are inverted, though not in direct spatial overlap (e.g., Wright, Gray, \& Diamond 2004).

Magnetic fields as strong as $40 \mathrm{mG}$ are seen in $\mathrm{OH}$ masers (Slysh \& Migenes 2006; Fish \& Reid 2007). Magnetic fields are highly ordered in star forming regions (Fish \& Reid 2006) and support pictures in which the processes of star formation do not tangle field lines significantly. While magnetic field strengths are usually stable from epoch to epoch, monotonic decay of the field in a Zeeman group in Cep A continues to be observed (Bartkiewicz et al. 2005). High spectral resolution observations support the conventional assumption that the Zeeman splitting coefficient appropriate for $\sigma^{ \pm 1}$ components should be assumed when measuring magnetic fields at 1612 and $1720 \mathrm{MHz}$ (Fish, Brisken, \& Sjouwerman 2006c). Linear polarization is of limited usefulness in determining the full, three-dimensional orientation of the magnetic field, likely due to a combination of Faraday rotation and anisotropic magnetohydronamic turbulence (Watson et al. 2004; Fish \& Reid 2006).

Extreme variability is occasionally seen in $\mathrm{OH}$. The $1665 \mathrm{MHz}$ maser in W75 N briefly flared to nearly $1 \mathrm{kJy}$ to become the brightest $\mathrm{OH}$ maser in the sky (Alakoz et al. 2005). 
The $4765 \mathrm{MHz}$ transition is highly time-variable (Palmer et al. 2004): the maser in Mon R2 flared to nearly 80 Jy before disappearing (Smits 2003) and reappearing (Fish et al. 2006b). Short-timescale variability is seen in the ground-state lines (Ramachandran, Deshpande, \& Goss 2006; Goss et al. 2007).

\subsection{Formaldehyde $\left(\mathrm{H}_{2} \mathrm{CO}\right)$}

Formaldehyde masers are seen near a handful of several massive YSOs, with several new detections in recent years (Araya et al. 2005, 2006). They have both a compact and an extended component with velocity gradients (Hoffman et al. 2003; Hoffman, Goss, \& Palmer 2007). A short-duration flare has been detected toward one source (Araya et al. 2007a). Further details can be found in the review on formaldehyde masers (Araya et al. $2007 b)$.

\subsection{Silicon monoxide (SiO)}

While SiO masers are commonly seen in evolved stars, they are rare in star forming regions. They are seen in bipolar outflows in W51 IRS 2 and Orion KL source I and appear much closer to the central source than do water masers (Eisner et al. 2002; Greenhill et al. 2004). As is the case in evolved stars, the maser species $\mathrm{SiO}$, water, and $\mathrm{OH}$ occur at progressively larger distances from source I (Cohen et al. 2006). While OH masers are ubiquitous throughout Orion, there is a "zone of avoidance" associated with source I in which they do not appear but $\mathrm{SiO}$ and water masers do. Interestingly, the $v=1, J=2 \rightarrow 1$ masers are found closer to the protostar in source I than are the $J=1 \rightarrow 0$ masers, a finding that is difficult to understand in the context of $\mathrm{SiO}$ maser pumping models (Doeleman et al. 2004).

\subsection{Other species}

Few other new maser species or transitions have been reported in the literature since the last meeting. The first $(J, K)=(6,6)$ ammonia $\left(\mathrm{NH}_{3}\right)$ maser has been detected centered on a millimeter peak in NGC 6334 I (Beuther et al. 2007). Weakly inverted acetaldehyde $\left(\mathrm{CH}_{3} \mathrm{CHO}\right)$ has been detected in the $1_{11} \rightarrow 1_{10}$ transition at $1065.075 \mathrm{MHz}$ toward Sgr B2 (Chengalur \& Kanekar 2003).

\section{Multi-species associations}

Methanol, $\mathrm{OH}$, and water masers are frequently in the same source, although water and methanol masers usually originate in different regions (Beuther et al. 2002; Caswell 2004b; Edris et al. 2005; Szymczak, Pillai, \& Menten 2005). Most $6.7 \mathrm{GHz}$ methanol maser sources have associated $\mathrm{OH}$ masers, almost always at $1665 \mathrm{MHz}$ and frequently at $1667 \mathrm{MHz}$ as well (Szymczak \& Gérard 2004), while the correlation between $6.7 \mathrm{GHz}$ methanol masers and $22 \mathrm{GHz}$ water masers is less strong (e.g., Breen et al. 2007). The distributions of $6.7 \mathrm{GHz}$ methanol masers and $6.0 \mathrm{GHz} \mathrm{OH}$ masers in $\mathrm{W} 3(\mathrm{OH})$ are very similar, although direct overlap of the two species is rare (Etoka et al. 2005). Similar phenomena are also seen between $6.7 \mathrm{GHz}$ methanol and 1.6/4.7 $\mathrm{GHz} \mathrm{OH}$ masers (Harvey-Smith \& Cohen 2006).

All three species correlate more strongly with mid infrared emission than centimeter or near infrared emission, and all are frequently found in linear groupings (De Buizer et al. 2005). However, the luminosity of water masers correlates less strongly with far infrared luminosity than is the case for methanol and $\mathrm{OH}$ masers, likely because water masers are not predominantly pumped by infrared photons (Szymczak et al. 2005), although they may not be pumped entirely by collisions either (Liu, Forster, \& Sun 2005; Liu 
et al. 2007). In any case, the existence of either masers (water and methanol) or outflows towards a UCH II region is an excellent predictor of the other (Codella et al. 2004), indicating that both masers and outflows are usually detectable in the UCH II phase.

\section{Observational advances}

\subsection{Proper motions and geometric distances}

Evidence in support of the kinematic interpretation of maser motions continues to pile up, with reports of the persistence of spot shapes in methanol (Moscadelli et al. 2002) and water masers (Goddi et al. 2006) and the inferred average overdensity of masers as compared to non-masing material (Fish, Reid, \& Menten 2005; Fish et al. 2006a). In addition to tracing internal source motions, maser proper motions can be used to obtain geometric parallax distances and measurements of Galactic rotation. Recent years have seen this technique used for both methanol and water masers in $\mathrm{W} 3(\mathrm{OH})$ to obtain distances accurate to a few percent ( $\mathrm{Xu}$ et al. 2006; Hachisuka et al. 2006). Further details can be found in proceedings in the Galactic structure session.

\subsection{Spectral resolution}

Very high spectral line observations at VLBI (very long baseline interferometry) spatial resolution have been obtained toward masers in several species in star forming regions. Detailed line profile analyses of water masers conclude that the near-Gaussian lineshapes indicate that they occur in hot $(\sim 1200 \mathrm{~K})$ gas with small beaming angles (Watson, Sarma, \& Singleton 2002). Similar observations of $12.2 \mathrm{GHz}$ methanol masers (Moscadelli et al. 2003) and the ground-state quartet of $\mathrm{OH}$ in W3(OH) (Fish et al. 2006c) find similarly Gaussian spectral profiles as well as maser spot positional gradients as a function of velocity (equivalently, velocity gradients). The positional gradients show no clear largescale spatial organization but have similar magnitudes in methanol and three of the four $\mathrm{OH}$ transitions. It is possible that these positional gradients represent turbulent motions on very small spatial scales. If so, it is important to understand the characteristics of the turbulence, since observed maser properties, including variability, can be highly sensitive to turbulence in the masing region (Böger, Kegel, \& Hegmann 2003; Sobolev, Watson, \& Okorokov 2003; Silant'ev et al. 2006). Similar polarization characteristics in Class II methanol features at different velocities may indicate that velocity gradients induce velocity redistribution (Wiesemeyer et al. 2004), which may play a critical role in preventing saturated rebroadening (e.g., Nedoluha \& Watson 1988).

\subsection{Infrared pumping lines}

Molecular infrared transitions observations, particularly of $\mathrm{OH}$, are essential for measuring maser pump efficiencies and may help place observational constraints on the radiative pump cycles of some models (e.g., Gray 2007). Archival Infrared Space Observatory data have been searched for the 34.6 and $53.3 \mu \mathrm{m}$ pumping lines of $\mathrm{OH}$ with limited success, due to the low spectral resolution of the instruments (He \& Chen 2004; He 2005). Herschel and SOFIA will have the spectral resolution and frequency coverage required to observe pumping lines of $\mathrm{OH}$ as well as the critical $560 \mu \mathrm{m}$ line of methylidyne $(\mathrm{CH})$.

\section{Further remarks}

Two quotes from De Buizer et al. (2005) serve to summarize the common themes of the observations over the past five years. The first is that "maser emission in general can trace a variety of phenomena associated with massive stars including shocks, outflows, 
infall, and circumstellar disks. No one maser species is linked exclusively to one particular process or phenomenon." Indeed, while certain maser species may preferentially turn up in a particular context (possibly a result of observational biases), the set of all observations of any one maser species resist being pigeonholed into a particular phenomenon. The second quote is that water, $\mathrm{OH}$, and methanol masers "do not seem to be associated with different early evolutionary stages of massive stars. Instead it appears that they all trace a variety of stellar phenomena throughout many early stages of massive stellar evolution." As is clear from $§ 3$, different maser species are commonly found together, independent of the evolutionary stage of the source. While proposed sequences in which certain masers turn on before others may be useful for statistical evaluation of evolutionary phases, important exceptions to such sequences exist. Those oddball masers that do not seem to fit present standard paradigms should be studied in especial detail, since we cannot predict beforehand what will be thereby learned about their environment or about maser processes in general.

It was only a few years ago that Ellingsen (2004) referred to masers as "the Bart Simpson of star formation research," noting that they are "under-achievers" in comparison with masers in other environments due to the lack of sensitive, high resolution observations at complementary wavelengths. While this may once have been true, recent maser observations have made great advancements in probing a wide range of dynamic structures relevant to star formation. Maser VLBI allows observations of small- and large-scale morphologies, magnetic fields, and motions on AU scales and is showing great promise as a tool to trace Galactic structure. Maser models for some species are becoming sufficiently refined to provide good constraints on physical conditions. The community is beginning to appreciate the role of turbulence and the ability to probe its properties using maser observations. Synergies with mid infrared instruments have clarified many of the mysteries of linear structures with velocity gradients. We have entered the era of greatly improved far infrared instrumentation, and the ALMA (Atacama Large Millimeter Array) era, with unprecedented sensitivity and angular resolution at submillimeter wavelengths, will begin in a few years. Further advancements in radio instrumentation, including new space VLBI missions and the SKA (Square Kilometre Array), will provide even greater insights. It is perhaps more correct to state that maser observations are at the vanguard of star formation research: yesterday's observations can be explained by complementary data and theory today, and today's observations lay the groundwork for the breakthroughs that will be achieved in the context of tomorrow.

\section{Acknowledgements}

The National Radio Astronomy Observatory is a facility of the National Science Foundation operated under cooperative agreement by Associated Universities, Inc.

\section{References}

Alakoz, A. V., Slysh, V. I., Popov, M. V., \& Val'tts, I. E. 2005, Astron. Lett., 31, 375

Araya, E., Hofner, P., Goss, W. M., Kurtz, S., Linz, H., \& Olmi, L. 2006, ApJ, 643, L33

Araya, E., Hofner, P., Kurtz, S., Linz, H., Olmi, L., Sewilo, M., Watson, C., \& Churchwell, E. 2005, ApJ, 618, 339

Araya, E., Hofner, P., Sewilo, M., Linz, H., Kurtz, S., Olmi, L., Watson, C., \& Churchwell, E. 2007a, ApJ, 654, L95

Araya, E., et al., 2007b, (these proceedings)

Argon, A. L., Reid, M. J., \& Menten, K. M. 2003, ApJ, 593, 925

Babkovskaia, N., \& Poutanen, J. 2004, A\&A, 418, 117

Bartkiewicz, A., Szymczak, M., Cohen, R. J., \& Richards, A. M. S. 2005, MNRAS, 361, 623 
Bartkiewicz, A., Szymczak, M., \& van Langevelde, H. J. 2005, A $\mathscr{E} A$, 442, L61

Baudry, A., \& Desmurs, J. F. 2002, A\& A, 394, 107

Beuther, H., Thorwith, S., Zhang, Q., Hunter, T. R., Megeath, S. T., Walsh, A. J., \& Menten, K. M. 2005, ApJ, 627, 834

Beuther, H., Walsh, A., Schilke, P., Sridharan, T. K., Menten, K. M., \& Wyrowski, F. 2002, $A \& A, 390,289$

Beuther, H., Walsh, A. J., Thorwith, S., Zhang, Q., Hunter, T. R., Megeath, S. T., \& Menten, K. M. 2007, $A \& A$, in press, astro-ph/0702190

Błaszkiewicz, L., \& Kus, A. J. 2004, A\&̈A, 413, 233

Böger, R., Kegel, W. H., \& Hegmann, M. 2003, A\& A, 406, 23

Breen, S. L., et al. 2007, MNRAS, in print, astro-ph/0702673

Caswell, J. L. 2003, MNRAS, 341, 551

Caswell, J. L. 2004a, MNRAS, 349, 99

Caswell, J. L. 2004b, MNRAS, 351, 279

Caswell, J. L. 2004c, MNRAS, 352, 101

Chengalur, J. N., \& Kanekar, N. 2003, A\& $A$, 403, L43

Codella, C., Lorenzani, A., Gallego, A. T., Cesaroni, R., \& Moscadelli, L. 2004, A\& A, 417, 615

Cohen, R. J., Gasiprong, N., Meaburn, J., \& Graham, M. F. 2006, MNRAS, 367, 541

Cragg, D. M., Sobolev, A. M., Caswell, J. L., Ellingsen, S. P., \& Godfrey, P. D. 2004, MNRAS, 351,1327

Cragg, D. M., Sobolev, A. M., \& Godfrey, P. D. 2005, MNRAS, 360, 533

De Buizer, J. M. 2003, MNRAS, 341, 277

De Buizer, J. M. 2006, ApJ, 642, L57

De Buizer, J. M. 2007, ApJ, 654, L147

De Buizer, J. M., \& Minier, V. 2005, ApJ, 628, L151

De Buizer, J. M., Radomski, J. T., Telesco, C. M., \& Piña, R. K. 2005, ApJS, 156, 179

De Buizer, J. M., Redman, R., Feldman, P., Longmore, S., \& Caswell, J. 2006, BAAS, 38, 1059

de Gregorio-Monsalvo, I., Gómez, J. F., Suárez, O., Kuiper, T. B. H., Anglada, G., Patel, N. A., \& Torrelles, J. M. 2006, AJ, 132, 2584

Dodson, R. G., \& Ellingsen, S. P. 2002, MNRAS, 333, 307

Dodson, R., Ojha, R., \& Ellingsen, S. P. 2004, MNRAS, 351, 779

Doeleman, S. S., Lonsdale, C. J., Kondratko, P. T., \& Predmore, C. R. 2004, ApJ, 607, 361

Edris, K. A., Fuller, G. A., \& Cohen, R. J. 2007, A\&SA, 465, 865

Edris, K. A., Fuller, G. A., Cohen, R. J., \& Etoka, S. 2005, A 8 A, 434, 213

Eisner, J. A., Greenhill, L. J., Herrnstein, J. R., Moran, J. M., \& Menten, K. M. 2002, ApJ, 569,334

Elitzur, M. 2002, Astrophysical Spectropolarimetry, 225

Ellingsen, S. P. 2004, IAU Symposium, 221, 133

Ellingsen, S. P. 2005, MNRAS, 359, 1498

Ellingsen, S. P. 2006, ApJ, 638, 241

Ellingsen, S. P. 2007, MNRAS, in press, astro-ph/0702506

Ellingsen, S. P., Cragg, D. M., Lovell, J. E. J., Sobolev, A. M., Ramsdale, P. D., \& Godfrey, P. D. 2004, MNRAS, 354, 401

Ellingsen, S. P., Cragg, D. M., Minier, V., Muller, E., \& Godfrey, P. D. 2003, MNRAS, 344, 73

Etoka, S., Cohen, R. J., \& Gray, M. D. 2005, MNRAS, 360, 1162

Fish, V. L., Brisken, W. F., \& Sjouwerman, L. O. 2006c, ApJ, 647, 418

Fish, V. L., \& Reid, M. J. 2006, ApJS, 164, 99

Fish, V. L., \& Reid, M. J. 2007, ApJ, 656, 943

Fish, V. L., Reid, M. J., Argon, A. L., \& Zheng, X.-W. 2005, ApJS, 160, 220

Fish, V. L., Reid, M. J., \& Menten, K. M. 2005, ApJ, 623, 269

Fish, V. L., Reid, M. J., Menten, K. M., \& Pillai, T. 2006a, A\&SA, 458, 485

Fish, V. L., Zschaechner, L. K., Sjouwerman, L. O., Pihlström, Y. M., \& Claussen, M. J. 2006b, ApJ, 653, L45

Furuya, R. S., Kitamura, Y., Wootten, A., Claussen, M. J., \& Kawabe, R. 2005, A\&A, 438, 571

Gallimore, J. F., Cool, R. J., Thornley, M. D., \& McMullin, J. 2003, ApJ, 586, 306 
Gillis, R. G., Pratap, P., \& Strelnitski, V. 2005, BAAS, 37, 1472

Goddi, C., \& Moscadelli, L. 2006, A\&A, 447, 577

Goddi, C., Moscadelli, L., Torrelles, J. M., Uscanga, L., \& Cesaroni, R. 2006, A\&\&A, 447, L9

Goedhart, S., Gaylard, M. J., \& van der Walt, D. J. 2003, MNRAS, 339, L33

Goedhart, S., Gaylard, M. J., \& van der Walt, D. J. 2005, Ap\&SSS, 295, 197

Goedhart, S., Minier, V., Gaylard, M. J., \& van der Walt, D. J. 2005, MNRAS, 356, 839

Gómez, de Gregorio-Monsalvo, I., Suárez, O., \& Kuiper, T. B. H. 2006, AJ, 132, 1322

Goss, W. M., et al., 2007, (these proceedings)

Gray, M. D. 2007, MNRAS, 375, 477

Greenhill, L. J., Reid, M. J., Chandler, C. J., Diamon, P. J., \& Elitzur, M. 2004, IAU Symp. 221,155

Hachisuka, K., Brunthaler, A., Menten, K. M., Reid, M. J., Imai, H., Hagiwara, Y., Miyoshi, M., Horiuchi, S., \& Sasao, T. 2006, ApJ, 645, 337

Harvey-Smith, L., \& Cohen, R. J. 2005, MNRAS, 356, 637

Harvey-Smith, L., \& Cohen, R. J. 2006, MNRAS, 371, 1550

He, J. H. 2005, New Astron., 10, 283

He, J. H., \& Chen, P. S. 2004, New Astron., 9, 545

Healy, K. R., Hester, J. J., \& Claussen, M. J. 2004, ApJ, 610, 835

Hoffman, I. M., Goss, W. M., \& Palmer, P. 2007, ApJ, 654, 971

Hoffman, I. M., Goss, W. M., Palmer, P., \& Richards, A. M. S. 2003, ApJ, 598, 1061

Hoffmann, S., Pratap, P., \& Strelnitski, V. 2006, BAAS, 38, 947

Honma, M., Bushimata, T., \& Choi, Y. K., et al. 2005, PASJ, 57, 595

Honma, M., Choi, Y. K., \& Bushimata, T., et al. 2004, PASJ, 56, L15

Hutawarakorn, B., \& Cohen, R. J. 2005, MNRAS, 357, 338

Imai, H., Horiuchi, S., Deguchi, S., \& Kameya, O. 2003, ApJ, 595, 285

Imai, H., Watanabe, T., Omodaka, T., Nishio, M., Kameya, O., Miyaji, T., \& Nakajima, J. 2002, PASJ, 54, 741

Lekht, E. E., Munitsyn, V. A., \& Tolmachev, A. M. 2005, Astron. Lett., 31, 315

Lekht, E. E., Silant'ev, N. A., Krasnov, V. V., \& Munitsyn, V. A. 2006, Astron. Rep., 50, 638

Lekht, E. E., Trinidad, M. A., Mendoza-Torres, J. E., Rudnitskij, G. M., \& Tolmachev, A. M. 2006, A\& A, 456, 145

Lemonias, J. J., Strelnitski, V., \& Pratap, P. 2006, BAAS, 38, 947

Liu, H., Forster, J. R., Liu, Y., \& Sun, J. 2007, ApSS, in press

Liu, H.-P., Forster, J. R., \& Sun, J. 2005, Chin. J. Astron. Astrophys., 5, 175

Menten, K. M., \& van der Tak, F. F. S. 2004, $A \mathscr{\mho} A$, 414, 289

Minier, V., \& Booth, R. S. 2002, A\&A, 387, 179

Minier, V., Booth, R. S., Ellingsen, S. P., Conway, J. E., \& Pestalozzi, M. R. 2000, EVN Sympsosium, Proceedings of the 5th European VLBI Network Symposium, 179

Minier, V., Burton, M. G., Hill, T., Pestalozzi, M. R., Purcell, C. R., Garay, G., Walsh, A. J., \& Longmore, S. 2005, A $\mathcal{E} A, 429,945$

Minier, V., Ellingsen, S. P., Norris, R. P., \& Booth, R. S. 2003, A\&A, 403, 1095

Moscadelli, L., Cesaroni, R., \& Rioja, M. J. 2005, A\&A, 438, 889

Moscadelli, L., Menten, K. M., Walmsley, C. M., \& Reid, M. J. 2002, ApJ, 564, 813

Moscadelli, L., Menten, K. M., Walmsley, C. M., \& Reid, M. J. 2003, ApJ, 583, 776

Moscadelli, L., Testi, L., Furuya, R. S., Goddi, C., Claussen, M., Kitamura, Y., \& Wootten, A. 2006, A\&A, 446, 985

Müller, H. S. P., Menten, K. M., \& Mäder, H. 2004, A\&A, 428, 1019

Norris, R. P., et al. 1998, ApJ, 508, 275

Nammahachak, S., Asanok, K., Hutawarakorn Kramer, B., Cohen, R. J., Muanwong, O., \& Gasiprong, N. 2006, MNRAS, 371, 619

Nedoluha, G. E., \& Watson, W. D. 1988, ApJ, 335, L19

Niezurawska, A., Szymczak, M., Cohen, R. J., \& Richards, A. M. S. 2004, MNRAS, 350, 1409

Niezurawska, A., Szymczak, M., Richards, A. M. S., \& Cohen, R. J. 2005, Ap\&SSS, 295, 37

Norris, R. P., Whiteoak, J. B., Caswell, J. L., Wieringa, M. H., \& Gough, R. G. 1993, ApJ, 412, 222 
Palmer, P., \& Goss, W. M. 2005, MNRAS, 360, 993

Palmer, P., Goss, W. M., \& Devine, K. E. 2003, ApJ, 599, 324

Palmer, P., Goss, W. M., \& Whiteoak, J. B. 2004, MNRAS, 347, 1164

Pashchenko, M. I., \& Lekht, E. E. 2005, Astron. Rep., 49, 624

Pestalozzi, M. R., Chrysostomou, A., Collett, J. L., Minier, V., Conway, J., \& Booth, R. S. 2007, $A \mathscr{E} A, 463,1009$

Pestalozzi, M. R., Elitzur, M., Conway, J. E., \& Booth, R. S. 2004, ApJ, 603, L113

Pestalozzi, M. R., Minier, V., \& Booth, R. S. 2005, A\&A, 432, 737

Pestalozzi, M. R., Minier, V., Motte, F., \& Conway, J. E. 2006, A\& $\&$, 448, L57

Phillips, C. J., \& van Langevelde, H. J. 2005, ApEESS, 295, 225

Pillai, T., Wyrowski, F., Menten, K. M., \& Krügel, E. 2006, A\&A, 447, 929

Pratap, P., Hoffmann, S., \& Strelnitski, V. 2006, BAAS, 38, 948

Ramachandran, R., Deshpande, A. A., \& Goss, W. M. 2006, ApJ, 653, 1314

Ripman, B. H., \& Strelnitski, V. 2006, BAAS, 38, 1053

Salii, S. V., \& Sobolev, A. M. 2006, Astron. Rep., 50, 965

Sarma, A. P., Troland, T. H., Crutcher, R. M., \& Roberts, D. A. 2002, ApJ, 580, 928

Seth, A. C., Greenhill, L. J., \& Holder, B. P. 2002, ApJ, 581, 325

Silant'ev, N. A., Lekht, E. E., Mendoza-Torres, J. E., \& Rudnitskij, G. M. 2006, A\&SA, 453, 989

Slysh, V. I., \& Migenes, V. 2006, MNRAS, 369, 1497

Slysh, V. I., Migenes, V., Val'tts, I. E., Lyubchenko, S.Yu., Horiuchi, S., Altunin, V. I., Fomalont, E. B., \& Inoue, M. 2002, ApJ, 564, 317

Slysh, V. I., Voronkov, M. A., Val'tts, I. E., \& Migenes, V. 2002, Astron. Rep., 46, 969

Smits, D. P. 2003, MNRAS, 339, 1

Sobolev, A. M., Watson, W. D., \& Okorokov, V. A. 2003, ApJ, 590, 333

Stark, D. P., Goss, W. M., Churchwell, E., Fish, V. L., \& Hoffman, I. M. 2007, ApJ, 656, 943

Strelnitski, V., et al., 2007, (these proceedings)

Strelnitski, V., Alexander, J., Gezari, S., Holder, B. P., Moran, J. M., \& Reid, M. J. 2002, ApJ, 581,1180

Sutton, E. C., Sobolev, A. M., Salii, S. V., Malyshev, A. V., Ostrovskii, A. B., \& Zinchenko, I. I. 2004, $A p J, 609,231$

Szymczak, M., \& Gérard, E. 2004, A\& $A$, 414, 235

Szymczak, M., Pillai, T., \& Menten, K. M. 2005, A\&A, 434, 613

Torrelles, J. M., Patel, N. A., Anglada, G., et al. 2003, ApJ, 598, L115

Urquhart, J. S., Thompson, M. A., Morgan, L. K., \& White, G. J. 2006, A\& $A$, 450, 625

Uscanga, L., Cantó, J., Curiel, S., Anglada, G., Torrelles, J. M., Patel, N. A., Gómez, J. F., \& Raga, A. C. $2005, A p J, 634,468$

Valdettaro, R., Palla, F., Brand, J., \& Cesaroni, R. 2005, A\& A, 535, 540

van der Walt, J. 2005, MNRAS, 360, 153

Vlemmings, W. H. T. 2006, A\&SA, 445, 1031

Vlemmings, W. H. T., Diamond, P. J., van Langevelde, H. J., \& Torrelles, J. M. 2006, A\& A, 448,597

Voronkov, M. A., Austin, M. C., \& Sobolev, A. M. 2002, A\& A, 387, 310

Voronkov, M. A., Brooks, K. J., Sobolev, A. M., Ellingsen, S. P., Ostrovskii, A. B., \& Caswell, J. L. 2006, MNRAS, 373, 411

Voronkov, M. A., Sobolev, A. M., Ellingsen, S. P., \& Ostrovskii, A. B. 2005, MNRAS, 362, 995

Watson, W. D., Sarma, A. P., \& Singleton, M. S. 2002, ApJ, 570, L37

Watson, W. D., Wiebe, D. S., McKinney, J. C., \& Gammie, C. F. 2004, ApJ, 604, 707

Wiesemeyer, A., Thum, C., \& Walmsley, C. M. 2004, A\&SA, 428, 479

Wright, M. M., Gray, M. D., \& Diamond, P. J. 2004, MNRAS, 350, 1272

Xu, Y., Reid, M. J., Zheng, X. W., \& Menten, K. M. 2006, Science, 311, 54

Zapata, L. A., Rodríguez, L. F., Ho, P. T. P., Beuther, H., \& Zhang, Q. 2006, AJ, 131, 939 